\section{Prophylaxe zerebrovaskulärer Erkrankungen}

Wiebke Jansen, Hans-Christian Koennecke

Gefäßzentrum Berlin / Abteilung für Neurologie, Ev. Krankenhaus Königin Elisabeth Herzberge

psychoneuro 2005; 31 (5): 276-281

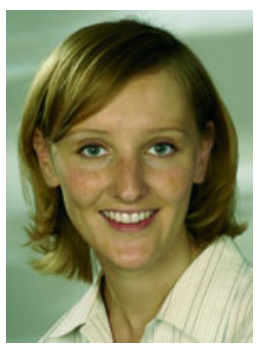

Wiebke Jansen
Angesichts der erwarteten Zunahme von Schlaganfällen aufgrund des künftig höheren Anteils älterer Menschen kommt der Prävention, auch unter gesundheitsökonomischen Aspekten, eine große Bedeutung zu. Die effektivste Form der Primärprävention besteht in der Behandlung von Risikofaktoren, insbesondere der arteriellen Hypertonie. Für die Sekundärprävention sind, neben der Behandlung von Risikofaktoren, vor allem antithrombotisch wirksame Medikamente relevant, wobei deren Indikation sich im Wesentlichen nach dem Pathomechanismus des stattgehabten Schlaganfalls richtet. Darüber hinaus lassen sich Rezidivereignisse durch revaskularisierende Maßnahmen an den hirnversorgenden Arterien vermeiden, vorausgesetzt die Indikation berücksichtigt die auf großen Studien basierende Evidenz.

. Deutschland erleiden jährlich 180000 bis 200000 Menschen einen Schlaganfall. In industrialisierten Ländern sind zerebrovaskuläre Erkrankungen die dritthäufigste Todesursache sowie die häufigste Ursache einer im Erwachsenenalter erworbenen, bleibenden Behinderung. Insoweit kommt ihrer Prävention auch eine erhebliche gesundheitsökonomische Bedeutung zu. Zudem ist aufgrund der mit dem Alter exponentiell steigenden Inzidenz und angesichts der demografischen Prognosen in den kommenden Jahrzehnten mit einer deutlichen Zunahme von Schlaganfällen zu rechnen.

Grundsätzlich basieren Primärund Sekundärprävention auf der Behandlung vaskulärer Risikofaktoren, antithrombotischer Therapie sowie revaskularisierenden Maßnahmen. In diesem Zusammenhang sollte begrifflich unterschieden werden zwi- schen den Risikofaktoren für Schlaganfälle einerseits und den unmittelbaren Auslösern zerebraler Ischämien andererseits. Wesentliche Risikofaktoren sind insbesondere arterielle Hypertonie, Diabetes mellitus, Zigarettenrauchen und vermutlich auch Fettstoffwechselstörungen, während zu den Auslösern, die letztlich Folge der Risikofaktoren sind, kardiale Emboliequellen, Stenosen der großen hirnversorgenden Arterien und Veränderungen an den kleinen Hirngefäßen (Atherosklerose und Lipohyalinose) zählen.

\section{Primärprävention}

Trotz erheblicher Fortschritte in der Sekundärprophylaxe des Schlaganfalls stellt die Primärprävention den effektivsten Ansatz zur Reduktion von Schlaganfällen dar, da sich hierdurch meist die für die Pathogenese von Schlaganfällen relevanten Veränderungen am Gefäßsystem verhindern lassen, welche zum Zeitpunkt eines Erstereignisses oftmals bereits irreversibel und mithin schwer behandelbar sind.

\section{Behandlung kardiovaskulärer Risikofaktoren}

Unter den behandelbaren Risikofaktoren des Schlaganfalls kommt der arteriellen Hypertonie die größte Bedeutung zu. Die präventive Effizienz einer Hochdruckbehandlung konnte in zahlreichen randomisierten Studien gezeigt werden, wobei bereits eine durchschnittliche Senkung um 6 mmHg die Schlaganfallinzidenz um mehr als $40 \%$ verringert (10). Zudem konnte gezeigt werden, dass auch ältere Menschen (> 70 Jahre), deren oftmals rein systolische Hypertonie lange als relativ harmlos galt, deutlich von einer antihypertensiven Behandlung profitieren (10). Diuretika, ACE-Hemmer, $\mathrm{AT}_{1}$-Antagonisten und Betablocker gelten als Mittel der ersten Wahl, wobei der therapeutische Effekt interindividuell sehr variabel ist und zwischen den verschiedenen Substanzklassen nur geringe Unterschiede hinsichtlich der präventiven Wirkung bestehen. Angesichts des mehr als 40\%-igen Anteils unbehandelter Hypertoniepatienten ist vielmehr die Empfehlung wichtig, die Hypertoniebehandlung konse- 
quent mit dem Ziel möglichst niedriger Blutdruckwerte und ggf. unter Zuhilfenahme von Experten durchzuführen. Ob Patienten mit asymptomatischen, hämodynamisch relevanten Stenosen hirnversorgender Arterien einem höheren Schlaganfallrisiko unter strikter antihypertensiver Behandlung ausgesetzt sind, ist derzeit unklar.

Patienten mit einem Diabetes mellitus haben ein deutlich erhöhtes Risiko insbesondere für ischämische Schlaganfälle. Leider konnte bisher nicht gezeigt werden, dass sich das Schlaganfallrisiko durch eine effektive antidiabetische Behandlung senken lässt, während die mikrovaskulären Komplikationen (Nephropathie, Retinopathie, Polyneuropathie, u.a.) hierdurch seltener den Verlauf komplizieren. Besonders wichtig zur Senkung des kardiovaskulären Risikos ist bei Diabetikern vielmehr die Behandlung einer in $40-60 \%$ der Fälle begleitenden Hypertonie, wobei der protektive Effekt des ACEHemmers Ramipril in diesem $\mathrm{Zu}-$ sammenhang am besten untersucht ist (8).

Während die Bedeutung einer Hyperlipidämie als Risikofaktor für Herzinfarkt und periphere arterielle Verschlusskrankheit (pAVK) unstrittig ist, ließ sich ein solcher Zusammenhang für den Schlaganfall bisher nicht herstellen. Demgegenüber jedoch bewirkt eine Therapie mit Statinen eine signifikante Reduktion des Schlaganfallrisikos (6), wobei dieser Effekt unabhängig von der rein cholesterinsenkenden Wirkung zu sein scheint und möglicherweise auf die antithrombotischen, antiinflammatorischen, neuroprotektiven und endothelialen Effekte von Statinen zurückzuführen ist. Bisher ließ sich dieser Effekt nur für ischämische Schlaganfälle unter einer Therapie mit Simvastatin nachweisen, während Pravastatin in verschiedenen Studien zu keiner signifikanten Reduktion der Schlaganfallhäufigkeit führte (9). Die Gabe von Simvastatin zur Primärprävention des Schlaganfalls kann daher insbesondere bei Patienten mit infolge kardiovaskulärer Komorbidität (Hypertonie, KHK, pAVK) erhöhtem Schlaganfallrisiko empfohlen werden.
Während eine starke Assoziation von erhöhten Homozysteinwerten und dem Schlaganfall-Risiko anhand zahlreicher Studien demonstriert werden konnte (2), steht der Nachweis eines kausalen Zusammenhangs bisher aus. Zudem ließ sich bisher nicht zeigen, dass mittels medikamentöser Behandlung einer Hyperhomozysteinämie durch Folat, Vitamin $B_{6}$ und $B_{12}$ die Inzidenz künftiger Schlaganfälle gesenkt wird, so dass bis zum definitiven Nachweis einer präventiven Wirkung vom unkritischen Einsatz dieser Substanzen abzuraten ist (5).

Inhalatives Zigarettenrauchen ist mit einer Verdopplung des Risikos ischämischer Schlaganfälle assoziiert. Da sich dieses Risiko rasch und deutlich (50\%) senken lässt, wenn das Rauchen aufgegeben wird, müssen Aufklärung und Motivierung feste Bestandteile der ärztlichen Maßnahmen zur Primärprävention sein. Gleiches gilt für die Motivierung zu körperlicher (sportlicher) Aktivität ( $\geq 30$ min pro Tag) und/ oder zur Gewichtsreduktion bei Adipositas, da sich hierdurch die mit einem erhöhten Schlaganfallrisiko assoziierten, komorbiden Bedingungen reduzieren lassen (4).

\section{Primärprophylaxe spontaner} zerebraler Blutungen

Etwa $10 \%$ aller Schlaganfälle in Deutschland sind intrazerebrale Blutungen (ICB), welche eine im Vergleich zum ischämischen Schlaganfall höhere Mortalität und Morbidität aufweisen. Unter den kardiovaskulären Risikofaktoren ist, ebenso wie bei zerebralen Ischämien, die arterielle Hypertonie der mit Abstand wichtigste, so dass deren $\mathrm{Be}$ handlung die effektivste Maßnahme zur Prävention von ICB darstellt (7). $\mathrm{Zu}$ den behandelbaren Risikofaktoren sind daneben Zigarettenrauchen sowie Alkoholkonsum zu zählen, mit deren Aufgabe sich das Risiko ebenfalls wirksam senken lässt. Zwischen 9\% und 14\% aller (dann oft als sekundär bezeichnete) ICB gelten mittlerweile als Folge einer antithrombotischen Therapie, wobei orale Antikoagulanzien eine führende Rolle einnehmen. Unabhängig von der Indikation steigt dabei das
Risiko einer ICB oberhalb eines INRWertes von 3,0 deutlich an. Höheres Alter und ausgeprägte mikroangiopathische, zerebrale Läsionen erhöhen in diesem Zusammenhang das Risiko zusätzlich.

Für lobäre Hirnblutungen (im Gegensatz zu den meist mit Hypertonie assoziierten Stammganglienblutungen) stellt die zerebrale Amyloidangiopathie (CAA) einen in seiner Bedeutung vermutlich unterschätzten Risikofaktor dar (7). Die CAA, welche zudem mit der Alzheimer-Erkrankung assoziiert ist, zeichnet sich durch eine Einlagerung von Amyloid in die Wand zerebraler Gefäße (kleine Arterien, Arteriolen und Kapillaren) aus. Leider ist diese Erkrankung bisher nur bioptisch in vivo verifizierbar. Auch existieren noch keine therapeutischen Konzepte zur Verhinderung von ICB bei Patienten mit CAA. Ob zudem die Gabe von antithrombotischen Medikamenten das ICB-Risiko bei Patienten mit CAA erhöht, ist ebenfalls unklar und kann allenfalls vermutet werden.

\section{Antithrombotische Behandlung zur Primärprävention zerebraler Ischämien}

Eine Primärprävention von ischämischen Schlaganfällen konnte für den Einsatz von Thrombozytenfunktionshemmern (nur ASS untersucht) bis vor kurzem nicht gezeigt werden. Allerdings wiesen mehrere große Studien eine 44\%-ige Risikoreduktion für Herzinfarkte nach, so dass der primärpräventive Einsatz von ASS v.a. bei älteren Patienten und solchen mit kardiovaskulären Risikofaktoren gerechtfertigt werden kann (2). Eine aktuelle, randomisierte und plazebokontrollierte Studie an fast 40000 Frauen über 45 Jahre, die niedrig dosiert ASS erhielten (100 mg jeden 2. Tag), konnte eine primärpräventive Wirkung auch für ischämische Schlaganfälle demonstrieren, ohne dass es zu einer signifikanten Zunahme zerebraler Blutungen kam (12). Die meisten Ereignisse ließen sich zudem bei Patientinnen $\geq 65$ Jahre verhindern. Bei asymptomatischer Karotisstenose wird die Gabe von ASS ebenfalls empfohlen, wobei hier der wesentliche 
Nutzen in einer Reduktion von Myokardinfarkten besteht (8). Daten zum Einsatz von Clopidogrel, Dipyridamol oder Ticlopidin in der Primärprävention des Schlaganfalls stehen nicht zur Verfügung.

Darüber hinaus besteht bei bestimmten kardialen Erkrankungen die Indikation zur primärpräventiven Antiaggregation oder Antikoagulation. Diesbezüglich sei auf den Beitrag von Reill und Darius in diesem Heft verwiesen.

Revaskularisierende Maßnahmen zur Primärprävention

In diesem Zusammenhang sind insbesondere Stenosen der extrakraniellen A. carotis interna (ACI) von Bedeutung, da für andere Gefäße (Vertebralarterien, intrakranielle Gefäße) keine validierten Therapiekonzepte existieren. Eine asymptomatische, $\geq 60 \%$-ige Stenose der ACI ist mit einem Schlaganfallrisiko von höchstens $2 \%$ pro Jahr assoziiert, wobei dies auch nicht-behindernde Insulte einschließt. Durch eine Operation (Thrombendarteriektomie) lässt sich dieses Risiko um die Hälfte auf $1 \%$ pro Jahr reduzieren, vorausgesetzt, das perioperative Komplikationsrisiko für einen Hirninfarkt liegt bei maximal 3\% (11). Dieses Komplikationsrisiko hängt zum einen ganz wesentlich von Geschick und Erfahrung des Operateurs ab. Zum anderen erhöhen zunehmendes Alter, kardiale Komorbidität (KHK, Herzinsuffizienz), kontralateraler Karotisverschluss sowie eine Restenose nach früherer Operation das Risiko. Möglicherweise stellt die Angioplastie mit Stenting eine Alternative zur Operation insbesondere bei Patienten mit erhöhtem OP-Risiko dar (16). Die Revaskularisation einer asymptomatischen Karotisstenose ist generell nur dann sinnvoll, wenn die Lebenserwartung des Patienten bei mindestens drei bis fünf Jahren liegt. Eine gepoolte Analyse der großen randomisierten Studien hat zudem gezeigt, dass der Benefit einer Karotisoperation bei Frauen wesentlich geringer ist (14). Unklar ist auch, ob Patienten mit im Verlauf progredienten Stenosen eher von einem Eingriff profitieren. Stenosen $\leq 60 \%$ stellen sicher keine Indikation zur Revaskula- risation dar. Prinzipiell sollte die Entscheidung zum Vorgehen im Einzelfall interdisziplinär unter Beteiligung von Neurologe, Interventionalist/Gefäßchirurg sowie ggf. Internist und Anästhesist erfolgen.

\section{Sekundärprävention}

Rezidivereignisse stellen eine wesentliche Bedrohung für Patienten nach einem stattgehabten Schlaganfall dar, wobei von einem Risiko von $15 \%$ nach zwei Jahren bzw. 30\% nach fünf Jahren auszugehen ist (8). Die Mortalität von Schlaganfall-Rezidiven verdoppelt sich gegenüber dem Erstereignis. Rezidive innerhalb von 30 Tagen verlaufen in bis zu 30\% der Fälle tödlich. Insgesamt ist das Risiko, ein Rezidiv zu erleiden, in den ersten Wochen und bei Patienten mit vaskulären Risikofaktoren sowie einer KHK oder pAVK am höchsten.

Zwischen 15\% und 20\% aller Hirninfarkte geht eine TIA voraus. In diesem Zusammenhang ist es besonders wichtig darauf hinzuweisen, dass Patienten nach einer TIA in 10-12\% einen Hirninfarkt innerhalb der nächsten drei Monate erleiden (davon $50 \%$ innerhalb von $48 \mathrm{~h}$ ), sofern keine präventiven Maßnahmen eingeleitet werden. Dies unterstreicht die Notwendigkeit einer sofortigen neurovaskulären Evaluation, um eine frühzeitige Sekundärprävention unter Berücksichtigung des zugrundeliegenden Pathomechanismus einzuleiten (2).

\section{Behandlung kardiovaskulärer Risikofaktoren}

Im Gegensatz zur Primärprävention liegen hierzu nur wenige Studien vor. Durch die Behandlung der arteriellen Hypertonie mit einer Kombination aus Diuretikum (Indapamid) und ACE-Hemmer (Perindopril) kann das Rezidivrisiko sowohl für ischämische Schlaganfälle als auch für Hirnblutungen deutlich gesenkt werden (relative Risikoreduktion [RRR] 44\%), wobei auch Patienten profitieren, die bereits mit anderen Medikamenten antihypertensiv behandelt werden (2). Es ist anzunehmen, dass andere Kombinationen aus ACE-Hemmer und Diuretikum in gleicher Weise wirksam sind.
Für Patienten mit Diabetes mellitus liegen bisher keine ausreichenden Daten vor, die einen sekundärpräventiven Effekt bei optimierter Diabetestherapie belegt hätten. Dennoch gibt es auch hier Hinweise, wonach Diabetiker offenbar in besonderer Weise von einer strikten antihypertensiven Behandlung (bei entsprechender Komorbidität) hinsichtlich kardiovaskulärer Ereignisse profitieren (8).

Trotz der protektiven Wirkung einer Statintherapie in der Primärprävention des Schlaganfalls konnte ein solcher Effekt für die Sekundärprävention bisher nicht eindeutig gezeigt werden, wird aber derzeit in einer großen Studie (SPARCL) untersucht. Die Gabe eines Statins (z.B. 40 mg Simvastatin/d) kann für Patienten nach Schlaganfall dennoch und unabhängig vom Lipidstatus bereits zum jetzigen Zeitpunkt empfohlen werden, da sie zu einer deutlichen Reduktion anderer kardiovaskulärer Ereignisse führt (6).

Auch wenn entsprechende Interventionsstudien fehlen, ist davon auszugehen, dass sich sowohl durch Beendigung von Zigarettenrauchen, als auch durch Gewichtsreduktion bei Adipositas eine Senkung des Schlaganfall-Rezidiv-Risikos erreichen lässt.

\section{Antithrombotische Therapie in der Sekundärprävention}

Unter den zahlreichen antithrombotisch wirkenden Substanzen ließ sich bisher ein sekundärpräventiver Effekt nur für Thrombozytenfunktionshemmer (TFH) und Vitamin-K-Antagonisten belegen (Tab. 1).

Für die Sekundärprävention nicht-kardioembolischer ischämischer Schlaganfälle sind TFH, allen voran ASS, Mittel der Wahl. Allerdings ist ihr Effekt vergleichsweise moderat: Anhand der bisher größten Metaanalyse konnte gezeigt werden, dass TFH das relative Risiko eines nicht-tödlichen Schlaganfalls um 23\% (absolut 2,5\%) reduzieren; bei Patienten mit begleitender KHK oder pAVK war die Risikoreduktion mit 31\% etwas höher (2). Zum Vergleich, die RRR durch orale Antikoagulation nach Schlaganfall in- 


\section{Tab. 1 Empfehlungen für die antithrombotische Sekundärprophylaxe bei zerebraler Ischämie}

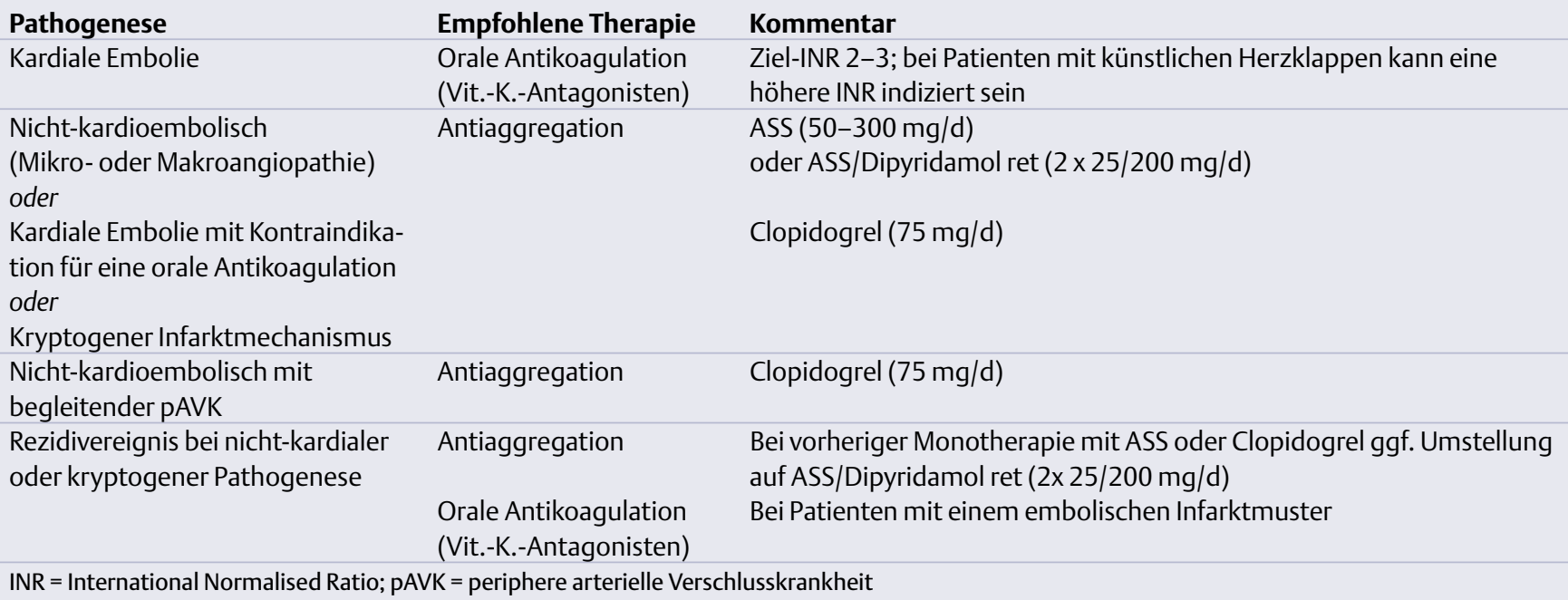

Empfohlene Therapie

Orale Antikoagulation

(Vit.-K.-Antagonisten)

Antiaggregation

Antiaggregation

Clopidogrel $(75 \mathrm{mg} / \mathrm{d})$

Bei vorheriger Monotherapie mit ASS oder Clopidogrel ggf. Umstellung auf ASS/Dipyridamol ret (2x 25/200 mg/d)

Bei Patienten mit einem embolischen Infarktmuster

INR = International Normalised Ratio; pAVK = periphere arterielle Verschlusskrankheit

\section{Kommentar}

Ziel-INR 2-3; bei Patienten mit künstlichen Herzklappen kann eine höhere INR indiziert sein

ASS (50-300 mg/d)

oder ASS/Dipyridamol ret $(2 \times 25 / 200 \mathrm{mg} / \mathrm{d})$

Clopidogrel (75 mg/d) usskrankheit

folge Vorhofflimmerns beträgt fast $70 \%$. Dennoch leisten TFH einen wesentlichen Beitrag zur Vermeidung von Hirninfarktrezidiven. Die Indikation zum primären Einsatz von ASS besteht prinzipiell nach zerebralen Ischämien infolge von Mikrooder Makroangiopathie sowie bei Patienten mit kardioembolischem Schlaganfall, die nicht antikoaguliert werden können. Gleiches gilt für ischämische Schlaganfälle unklarer Ätiologie (8). Hinsichtlich der Dosierung kann mittlerweile als gesichert gelten, dass sich Tagesdosen zwischen 50 und $325 \mathrm{mg}$ ASS nicht in ihrer präventiven Wirksamkeit unterscheiden (2).

Die kombinierte Antiaggregation mit niedrig dosiertem ASS ( 2 × 25 $\mathrm{mg}$ ) und retardiertem Dipyridamol (2 x $200 \mathrm{mg}$ ) erwies sich in einer großen Studie als überlegen im Vergleich zur Monotherapie mit ASS oder Dipyridamol, so dass diese Kombination als primäre Therapie zur Sekundärprävention eingesetzt werden kann (2).

Substanzen aus der Stoffgruppe der Thienopyridine (Clopidogrel und Ticlopidin) stellen eine Alternative zur Behandlung mit ASS dar. Clopidogrel ist in der Schlaganfallprävention etwa gleich wirksam wie ASS (1), weist jedoch ein etwas günstigeres Nebenwirkungsprofil auf. Dies allein rechtfertigt jedoch nicht den primären Einsatz von Clopidogrel beim nicht-kardio- embolischen Schlaganfall, zumal Clopidogrel um ein Vielfaches teurer als ASS ist. Bei Patienten mit pAVK erwies sich Clopidogrel allerdings als die gegenüber ASS effektivere Substanz, so dass der primäre Einsatz von Clopidogrel bei diesen $\mathrm{Pa}$ tienten nach Schlaganfall zu erwägen ist. Darüber hinaus ist es häufige klinische Praxis, Patienten, die unter einer ASS-Behandlung einen ischämischen Insult erlitten haben, auf Clopidogrel umzusetzen. Allerdings liegen keinerlei Studiendaten vor, die ein solches Vorgehen rechtfertigten. Ticlopidin, obwohl vermutlich sogar besser wirksam als ASS (8), wurde in den letzten Jahren weitgehend von Clopidogrel verdrängt, da es mit einer ca. 1\%-igen Inzidenz schwerer Neutropenien assoziiert ist. Dies sollte jedoch kein Grund sein, bereits langfristig mit Ticlopidin erfolgreich und ohne Nebenwirkungen behandelte Patienten prinzipiell auf ein anderes Präparat umzustellen.

Hinsichtlich intrakranieller Blutungen, der bedeutsamsten Nebenwirkung von TFH, sind keine wesentlichen Unterschiede zwischen den Substanzen bekannt (2). Für eine langfristige Kombinationsbehandlung mit ASS und Clopidogrel besteht in der Schlaganfallprävention jedoch keine Indikation, da unter dieser Therapie hämorrhagische Komplikationen gehäuft vorkommen (3).
Die Indikation zur Antikoagulation (AK) nach einem ischämischen Schlaganfall ist gegenwärtig allein bei Sicherung einer kardialen Emboliequelle (Vorhofflimmern, links-kardialer Thrombus) gegeben (s. Beitrag von Reill und Darius in diesem Heft). Für die meisten Indikationen (außer mechanische Herzklappen) ist eine Ziel-INR von 2-3 zu empfehlen. Es sei an dieser Stelle darauf hingewiesen, dass insbesondere ältere (> 75 Jahre) Patienten nach einer kardioembolischen zerebralen Ischämie einem Rezidivrisiko von bis zu 20\% pro Jahr ausgesetzt sind, so dass gewichtige Argumente vorliegen sollten, um sich gegen eine Antikoagulation zu entscheiden. Einzelne Stürze in der Anamnese sowie hohes Alter allein rechtfertigen ein Vorenthalten der AK sicher nicht. Auch sollte bei Zweifeln an der Compliance immer versucht werden, diese durch zusätzliche Maßnahmen wie Einbeziehung Angehöriger oder Anbindung an einen Pflegedienst zu gewährleisten.

Keine gesicherte Therapie gibt es bisher für Patienten mit ausgeprägten atherothrombotischen Veränderungen der proximalen Aorta ohne weitere mögliche Ursachen einer zerebralen Ischämie. Prinzipiell gilt derzeit, dass beim nichtkardioembolischen Hirninfarkt die ASS-Behandlung der oralen Antikoagulation ebenbürtig, jedoch mit weniger Blutungskomplikationen assoziiert ist (8). 


\section{Tab. 2 Empfehlungen für die Therapie bei Obstruktionen hirnversorgender Arterien}

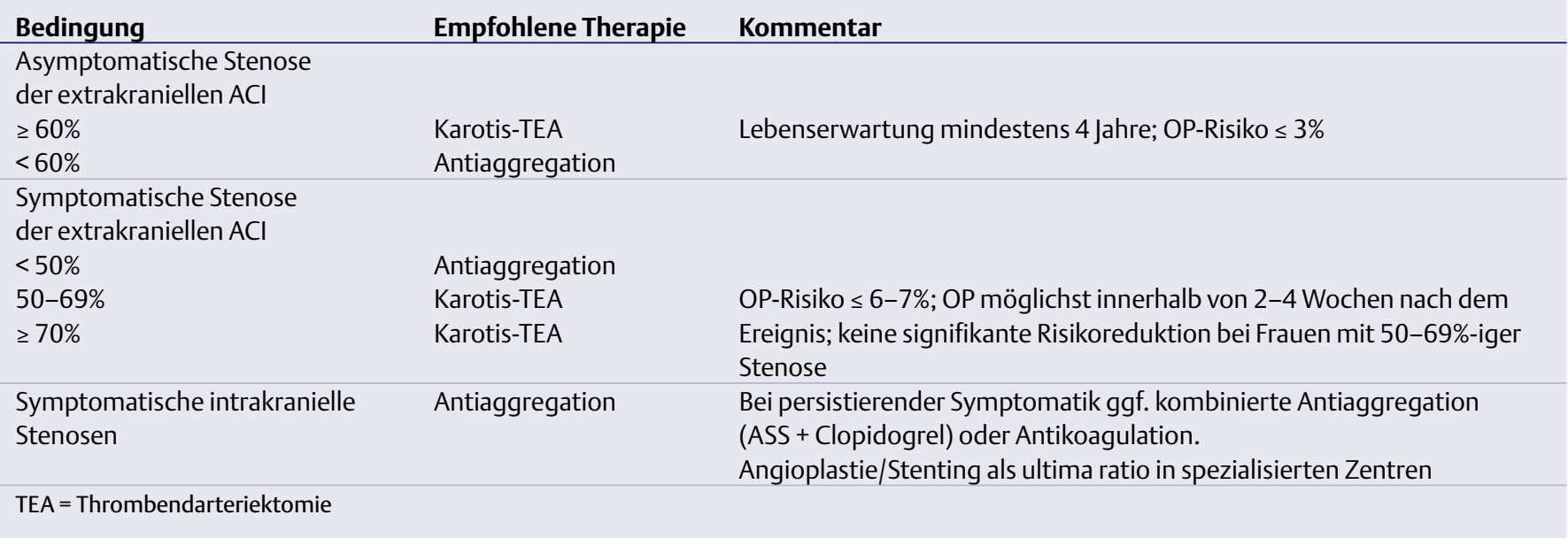

Revaskularisierende Maßnahmen zur Sekundärprävention

Der therapeutische Nutzen einer Operation (Tab. 2) ist bei Patienten mit hochgradiger (i.e. $\geq$ $70 \%$ ), symptomatischer Karotisstenose anhand großer, randomisierter Studien gut belegt (2). Das maximal zu tolerierende Komplikationsrisiko liegt dabei zwischen 6\% und $7 \%$. Ein höheres Risiko führt auch hier zu einer Aufhebung des potenziellen Nutzens einer Operation. Dieser Nutzen ist zudem abhängig vom Geschlecht des Patienten und dem zeitlichen Abstand zwischen der zerebralen Ischämie und dem Eingriff: Liegt das Ereignis (TIA oder nicht-behindernder Hirninfarkt) länger als zwei bis vier Wochen zurück, profitieren Frauen kaum noch, während bei Männern mit hochgradigen Stenosen der Eingriff selbst drei Monate und mehr nach einem zerebralen ischämischen Ereignis noch zu einer deutlichen Risikoreduktion führt (13). Bei nur mittelgradigen Stenosen (5069\%) ist der Benefit bei Männern mit kurz zurückliegendem Ereignis weiterhin evident, wohingegen die Nutzen-Risiko-Bilanz für Frauen keine Empfehlung zur Operation rechtfertigt (13). Ursächlich für die geschlechtsspezifischen Unterschiede sind vermutlich die einerseits höheren perioperativen Komplikationsraten bei Frauen, andererseits das bei konservativem Vorgehen gegenüber Männern niedrigere Hirninfarktrisiko (15).
Im Gegensatz zu asymptomatischen Patienten steigt bei symptomatischen Stenosen der Benefit einer Operation mit zunehmendem Alter (v.a. > 75 Jahre), so dass auch diese Patienten, trotz eines oft höheren perioperativen Risikos, einer revaskularisierenden Maßnahme zugeführt werden sollten. Auch hier stellt künftig möglicherweise die endovaskuläre Intervention mit Implantation eines Stents eine Alternative mit geringerem Risiko dar (16).

Auch bei der Therapieplanung für Patienten mit symptomatischen Karotisstenosen ist eine interdisziplinäre Zusammenarbeit anzustreben. Dabei spielt insbesondere die neurologische Evaluation eine bedeutende Rolle für die Klassifizierung einer Stenose als „symptomatisch“, da die korrekte Zuordnung der Symptomatik gerade bei transienten Störungen nicht immer einfach ist. Nicht jede „Sehstörung“ oder jedes „Kribbeln“, weder unspezifischer Schwindel, noch Synkopen sind Symptome einer Karotisstenose!

\section{Sekundärprophylaxe zerebraler Blutungen}

Hierzu liegen nur wenige Daten vor. Allerdings konnte die bisher größte Studie zu dieser Frage eine eindrucksvolle relative Risikoreduktion (50\%) durch eine antihypertensive Kombinationstherapie mit Indapamid und Perindopril nachweisen (8).
Mit Ausnahme von Patienten mit mechanischer Herzklappe stellt eine anamnestisch stattgehabte ICB i.d.R. eine Kontraindikation für den Einsatz antithrombotisch wirksamer Medikamente dar.

Ebenso ungelöst wie schwierig ist allerdings die Frage, ob dies auch für Patienten mit hohem Embolierisiko (etwa bei Vorhofflimmern oder links-kardialem Thrombus) und länger zurückliegender ICB gilt (7).

\section{Prophylaxis of cerebrovascular diseases} Given the rising proportion of elderly in future decades stroke incidence is likely to increase. Due to the enormous socioeconomic impact of stroke, its prevention is a matter of major significance. Primary prevention is primarily focused on and mostly effective through treatment of risk factors, among which hypertension is the most important. Besides risk factor treatment, several antithrombotic regimens are available for secondary prevention and need to be applied according to the underlying pathomechanism of cerebral ischemia. In addition, revascularization of stenosed carotid arteries may prevent further events, provided that they have been indicated appropriately according to the results of large trials.

\section{Key Words}

stroke - prevention - risk factors carotid surgery 


\section{Literatur}

1. CAPRIE Steering Committee. A randomised, blinded, trial of clopidogrel versus aspirin in patients at risk of ischaemic events. Lancet 1996; 348: 1329-39

2. Diener HC. Leitlinien der Deutschen Gesellschaft für Neurologie. Primäre und sekundäre Prävention der zerebralen Ischämie. 2003 http://www.dgn.org/127.0.html 3. Diener HC, Bogousslavsky J, Brass LM et al. on behalf of the MATCH Investigators: Aspirin and clopidogrel compared with clopidogrel alone after recent ischaemic stroke or transient ischaemic attack in high-risk patients (MATCH): randomised, double-blind, placebo-controlled trial. Lancet 2004; 364 : 331-337

4. Goldstein LB, Adams A, Becker K et al. Primary prevention of ischemic stroke. A statement for healthcare professionals from the stroke council of the American Heart Association. Circulation 2001; 103: 163182

5. Hankey G], Eikelboom JW. Homocystein and stroke (comment). Lancet 2005; 365: 194-196

6. Heart Protection Study Collaborative Group. Effects of cholesterol-lowering with simvastatin on stroke and other major vascular events in 20536 people with cerebrovascular disease or other high-risk conditions: a randomised placebo-controlled trial. Lancet 2004; 363: 757-767

7. Hug A, Aschoff A, Schwab S. Spontane intrazerebrale Blutungen. Nervenarzt 2004; 75: 953-961

8. Koennecke H-C. Secondary prevention of stroke. A practical guide to drug treatment. CNS Drugs 2004; 18: 221-241

9. Külkens S, Ringleb PA, Hacke W. Empfehlungen der European Stroke Initiative (EUSI) zur Behandlung des ischämischen Schlaganfalls - Aktualisierung 2003. Teil 2: Prävention und Rehabilitation. Nervenarzt 2004: 75: 380-388

10. Lawes CMM, Bennett DA, Feigin VL, Rodgers A. Blood pressure and stroke. An overview of published trials. Stroke 2004; 35: 1024-1033

11. MRC Asymptomatic Carotid Surgery Trial (ACST) Collaborative Group. Prevention of disabling and fatal strokes by successful carotid endarterectomy in patients without recent neurological symptoms: randomised controlled trial. Lancet 2004; 363: 14911502

12. Ridker PM, Cook NR, Lee IM et al. A randomized trial of low-dose aspirin in the primary prevention of cardiovascular disease in women. N Engl ] Med 2005; 352: 12931304

13. Rothwell PM, Eliasziw M, Gutnikow SA et al. Sex difference in the effect of time from symptoms to surgery on benefit from carotid endarterectomy for transient ischemic attack and non-disabling stroke. Stroke 2004; 35: 2855-2861

14. Rothwell PM, Goldstein LB. Carotid endarterectomy for asymptomatic carotid stenosis. Asymptomatic carotid surgery trial. Stroke 2004; 35: 2425-2427

15. Rothwell PM, Eliasziw M, Gutnikow SA et al. Endartectomy for symptomatic caro- tid stenosis in relation to clinical subgroups and timing of surgery. Lancet 2004: 363 : 915-924

16. Yadav JS, Wholey MH, Kuntz RE et al. for the Stenting and Angioplasty with Protection in Patients at High Risk for Endarterectomy Investigators. Protected carotid-artery stenting versus endarterectomy in high-risk patients. N Engl J Med 2004; 351: 1493-1501

Korrespondenzadresse

PD Dr. H.-C. Koennecke

Gefäßzentrum Berlin

Abteilung für Neurologie

Ev. Krankenhaus Königin Elisabeth Herzberge Herzbergstr. 79

10365 Berlin
TREVILOR $^{\star x}$ retard $75 \mathrm{mg} /$-retard $150 \mathrm{mg}$ Wirkstoff: Venlafaxinhydrochlorid Zusammensetzung: Eine Retardkapsel enthäl $84,84 \mathrm{mg}$ bzw. $169,7 \mathrm{mg}$ Venlafaxinhydrochlorid entsprechend $75 \mathrm{mg}$ bzw. $150 \mathrm{mg}$ Venlafaxin. Sonstige Bestandteile: Mikrokristalline Cellulose, Hy promellose, Ethylcellulose, Gelatine, Schellack, Propylenglycol, -Titandioxid (E 171), Eisen(III)-oxid (E 172), Eisenoxidhydrat (E 172); -retard $75 \mathrm{mg}$ zusätzlich: Simeticon; -retard $150 \mathrm{mg}$ zusätzlich: Povidon, Natriumhydroxid. Anwendungsgebiete: Depressive Erkrankungen, einschl. Depressionen mit begleitenden Angstzuständen. Erhaltungstherapie und Rezidivprophylaxe depressiver Erkrankungen. Generalisierte Angststörung, gekennzeichnet durch übermäßige Angst und Besorgnis bezüglich mehrerer Ereignisse und Tätigkeiten mit Begleitsymptomen wie Unruhe, leichte Ermüdbarkeit, Konzentrationsschwierigkeiten, Reizbarkeit, Muskelspannung oder Schlafstörungen. Soziale Angststörung (soziale Phobie) Gegenanzeigen: Kontraindiziert bei Patienten mit allergischen Reaktionen auf einen der Inhaltsstoffe, gleichzeitige Anwendung mit MAO-Hemmern (bei Wechsel auf bzw. von Venlafaxin 14 Tage Abstand, bei Wechsel von reversiblen MAO-Hemmern ggf. auch kürzer). Strengste Indikationsstellung in der Schwangerschaft (cave: Entzugserscheinungen beim Neugeborenen) und Stillzeit, ggf. Abstillen. Vorsichtsmaßnahmen: Nur unter besonderer Vorsicht anwenden bei: Patienten mit mäßiger bis schwerer Einschränkung der Leber- und Nierenfunktion oder Leberzirrhose; Patienten mit Krampfanfällen in der Anamnese; Patienten, die zuvor einer Elektroheilkrampfbehandlung unterzogen wurden; Dehydrierten oder Patienten mit Volumenmangel; Patienten mit kürzlich zurükkliegendem Herzinfarkt oder nicht stabilisierten Herzerkrankungen aufgrund mangelnder Erfahrung; Pat. deren Gesundheitszustand durch eine Erhöhung der Herzfrequenz beeinträchtigt werden könnte, insb. bei höheren Dosierungen. Die Anwendung bei Kindern und Jugendlichen unter 18 Jahren wird gegenwärtig nicht empfohlen, da hier die Wirksamkeit und Sicherheit bis jetzt nicht abgesichert ist; wenn es eingesetzt wird, dann regelmäßige Gewichts- und Blutdruckkontrolle. Sorgfältige Uberwachung bei: Patienten mit manischen Episoden in der Anamnese (Risiko manischer oder hypomanischer Zustände); Patienten mit erhöhtem Risiko zu akutem Engwinkelglaukom oder erhöhtem Augeninnendruck; Patienten mit Prädisposition zu Haut- und Schleimhautblutungen; Patienten mit Missbrauchs- und Abhängigkeitsanamnese. Bei Langzeitbehandlung sollte der Cholesterinspiegel bestimmt werden. Nebenwirkungen: Meis dosisabhängig und zu Beginn der Behandlung: Häufig Asthenie/Erschöpfung, gelegentlich Überempfindlichkeitsreaktionen gegenüber Licht, sehr selten Anaphylaxie. Häufig Blutdruckanstieg, Vasodilatation (meist Hitzewallungen). Gelegentlich Synkopen, Tachykardie, Hypotonie, orthostatische Hypotonie. Sehr selten QT- und QRS-Verlängerung, Kammerflimmern, ventrikuläre Tachykardie (einschl. Torsade de pointes). Gelegentlich kleinflächige Hautblutungen, Schleimhautblutungen; selten verlängerte Blutungsdauer, Thrombopenie. Berichte über weitere Blutbildveränderungen (Agranulozytose, aplastische Anämie, Neutropenie, Panzytopenie). Sehr häufig Übelkeit. Häufig Gähnen, Erbrechen, Appetitlosigkeit, Verstopfung, erhöhte Cholesterinwerte, Gewichtsabnahme. Ferner Berichte über pulmonale Eosinophilie. Gelegentlich Bruxismus, Leberwertveränderungen, Gewichtzunahme, Hyponatriämie. Berichte über Fälle von Pankreatitis und Diarrhoe. Selten Hepatitis, Syndrom inadäquater Ausschüttung des antidiuretischen Hormons (SIADH). Häufig Schwindel, Mund trockenheit, Schlaflosigkeit, Nervosität, Sedierung, ungewöhnliche Trauminhalte, Libidoabnahme, Parästhesien, Zittern, erhöhte Muskelspannung. Ferner Berichte über Prolaktinspiegel-Erhöhung, Rhabdomyolyse. Gelegentlich Apathie, Halluzinationen, Myoklonus, selten Krampfanfälle, Manie, malignes neuroleptisches Syndrom, Serotoninsyndrom. Ferner Berichte über Agitation, Delirium, extrapyramidale Reaktionen einschl. Dystonien, Dyskinesien, tardive Dyskinesien. Häufig Akkommodationsstörungen des Auges, Mydriasis, Sehstörungen, Schwitzen. Gelegentlich Geschmacksveränderungen, Alopezie, Hautausschlag, sowie Fälle von Tinnitus. Sehr selten Erythema multiforme, Steven-JohnsonSyndrom. Ferner wurde über Fälle von Pruritus berichtet. Häufig Ejakulations-, Orgasmus- und Potenzstörungen beim Mann, Probleme beim Wasserlassen. Gelegentlich Orgasmusstörungen bei der Frau, Menorrhagie, Harnverhaltung. Absetzerscheinungen mit Müdigkeit Schläfrigkeit, Schlafstörungen, Schlaflosigkeit, Kopfschmerzen, Tinnitus, zerebrale Krämpfe, Benommenheit, Nervosität, Ubelkeit, Schwindel, Angst, Verwirrtheit, Hypomanie, Parästhesien, Mundtrockenheit, Erbrechen und Durchfall, Erregung, Appetitlosigkeit, Schwitzen. In pädiatrischen klinischen Studien traten vermehrt Berichte über Feindseligkeit und, speziell be schwerer Depression, mit Suizid assoziierbare Ereignisse (wie Suizidgedanken und Selbstverletzung) auf. Wie bei Erwachsenen wurden verminderter Appetit, Gewichtsabnahme, Blutdruckanstieg und erhöhte Cholesterinwerte beobachtet. Außerdem wurden Bauchschmerzen, Agitiertheit, Dyspepsie, kleinflächige Hautblutungen, Nasenbluten und Myalgien beobachtet. Hinweise: Individuelle Einschränkungen des Reaktionsvermögen nicht ausschließbar, daher die Patienten entsprechend instruieren. Aus allgemeinen Vorsichtsgründen kein gleichzeitiger Alkoholgenuss. Regelmäßige Blutdruckkontrollen werden empfohlen. Engmaschige Kontrolle von Patienten unter Antikoagulantien-

Therapie. Verschreibungspflichtig.

Wyeth Pharma GmbH, 48136

Münster. Stand: Mai 2004 